\title{
Second Wave of COVID-19 Pandemic in Malaysia and the Impact on Nuclear Cardiology Services: An Institutional Experience
}

\author{
Ahmad Zaid Zanial ${ }^{1}$, Syifaa' Aminudin ${ }^{2}$, FatinHayani Mohamad Najib ${ }^{3}$, Siti \\ Zarina Amir Hassan ${ }^{1}$ \\ ${ }^{1}$ Nuclear Medicine Department, Hospital Kuala Lumpur, ${ }^{2}$ Pharmacy Department, Hospital \\ Kuala Lumpur, ${ }^{3}$ Nuclear Medicine Department, Hospital Kuala Lumpur \\ DOI: http://dx.doi.org/10.31344/ijhhs.v5i0.316
}

Introduction: Nuclear cardiology applying radioactive tracer and hybrid imaging techniques are able to provide information needed to detect and evaluate ischaemic heart diseases. In our centre, nuclear cardiology services involving stress and rest myocardial perfusion scans and viability studies contribute about $40 \%$ of overall scan workload. The second wave of COVID19 pandemic in Malaysia announced by the end of February 2020 has affected nuclear cardiology services.

Objectives: Our aims were to determine the impact of COVID-19 pandemic second wave on the nuclear cardiology imaging studies performed as well as to ascertain crucial institutional experience especially unavoidable problem and adjustment during the period.

Methods: A review of Technetium-99m tetrofosmin radiopharmaceutical dispensing data and scan records for 1st February to 31st August 2019 and 2020 was conducted at Nuclear Medicine Department, Hospital Kuala Lumpur. Figures were compiled and statistical analysis done. Survey and focus discussion conducted involving nuclear medicine physicians, pharmacists and physic officers to identify the main difficulty faced and most important Job-adapting measure taken.

Results: A total of 1109 cardiac radiopharmaceutical doses dispensed during the studied period but were less when compared to 1342 doses dispensed last year. Significant reduction was noted in April and May 2020 with only 69 and 67 cases respectively in comparison with monthly average of 192 cases in 2019. Although some scan appointments were postponed to avoid the usual waiting area congestions, the main difficulty faced was Technetium-99m generator supply disruption with limited production in Europe and international transportation restriction. Implementing infection control standard operating procedure (SOP) instructions as part of routine work practice with emphasis of time, distance and shielding concept was the most important Job-adapting measure.

Conclusion: Nuclear cardiology services were affected by the second wave of COVID-19 pandemic locally. Main problem ascertained was disruption of radioactive supply. Most important adjustment was infection control SOP implementation.

Keywords: COVID-19, pandemic, nuclear cardiology, experience 